\title{
BMJ Open Protocol for a systematic review of reporting standards of anaesthetic interventions in randomised controlled trials
}

\author{
Lucy Elliott (D) , ${ }^{1}$ Karen Coulman (D) , ${ }^{2}$ Natalie S Blencowe (D) , ${ }^{1}$ Mahim Qureshi, ${ }^{1}$ \\ Sethina Watson, ${ }^{3}$ Ronelle Mouton (D) , ${ }^{1,3}$ Robert J Hinchliffe (i) ${ }^{1,3}$
}

To cite: Elliott $\mathrm{L}$, Coulman $\mathrm{K}$, Blencowe NS, et al. Protocol for a systematic review of reporting standards of anaesthetic interventions in randomised controlled trials. BMJ Open 2020;10:e034372. doi:10.1136/ bmjopen-2019-034372

- Prepublication history and additional material for this paper are available online. To view these files, please visit the journal online (http://dx.doi. org/10.1136/bmjopen-2019034372).

Received 22 September 2019 Revised 03 December 2019 Accepted 18 December 2019

A) Check for updates

(C) Author(s) (or their employer(s)) 2020. Re-use permitted under CC BY-NC. No commercial re-use. See rights and permissions. Published by BMJ.

${ }^{1}$ Centre for Surgical Research, University of Bristol Medical School, Bristol, UK

${ }^{2}$ Population Health Sciences, University of Bristol Medical

School, Bristol, UK

${ }^{3}$ Southmead Hospital, North Bristol NHS Trust, Bristol, UK

Correspondence to

Dr Lucy Elliott;

lucy.elliott@nbt.nhs.uk

\section{ABSTRACT}

Introduction There is significant variation in how anaesthesia is defined and reported in clinical research. This lack of standardisation complicates the interpretation of published evidence and planning of future clinical trials. This systematic review will assess the reporting of anaesthesia as an intervention in randomised controlled trials (RCT) against the Consolidated Standards of Reporting Trials for Non-Pharmacological Treatments (CONSORT-NPT) framework.

Methods and analysis Online archives of the top six journals ranked by impact factor for anaesthesia and the top three general medicine and general surgery journals will be systematically hand searched over a 42-month time period to identify RCTs describing the use of anaesthetic interventions for any invasive procedure. All modes of anaesthesia and anaesthesia techniques will be included. All study data, including the type of anaesthetic intervention described, will be extracted in keeping with the CONSORT-NPT checklist. Descriptive statistics will be used to summarise general study details including types/modes of anaesthetic interventions, and reporting standards of the trials.

Ethics and dissemination No ethical approval is required. The results will be used to inform a funding application to formally standardise general, local, regional anaesthesia and sedation for use in clinical research. The systematic review will be disseminated via peer-reviewed manuscript and conferences.

PROSPERO registration number CRD42019141670.

\section{INTRODUCTION}

The choice of anaesthetic technique for different types of surgery and invasive procedures, and their suitability for individual patients, relies largely on evidence from highquality randomised controlled trials (RCT) and clinician expertise. Despite the widespread use of anaesthesia, there is significant variation in how it is defined and reported in clinical research and consensus definitions are lacking. ${ }^{1}$ For example, there is significant overlap between definitions of deep sedation and general anaesthesia (GA). ${ }^{1}$ The
Strengths and limitations of this study

- This will be the first systematic review to summarise the reporting of anaesthesia, to include general, local, regional anaesthesia and sedation as an intervention in randomised controlled trials (RCT) against the Consolidated Standards of Reporting Trials for Non-Pharmacological Treatments checklist.

- The findings from this systematic review will guide future research to improve the standardisation and reporting of anaesthetic interventions in clinical research.

- Only RCTs comparing anaesthetic interventions (eg, where anaesthesia is the main focus) will be included.

American Society of Anesthesiologists define GA as a 'drug-induced loss of consciousness during which patients are not arousable, even by painful stimulation, ${ }^{2}$ and sedation is defined by the Royal College of Anaesthetists as 'a continuum of depressed conscious state with unpredictable inter-individual dose responses to the drugs used, which may result in unconsciousness'. ${ }^{3}$ This may explain why standardised scales to routinely measure, record and standardise depth of sedation are lacking.

Clinician autonomy is acknowledged as a necessity in anaesthesia and is a fundamental reason for variation in practice. There is emerging evidence that the choice of mode of anaesthesia (GA, local anaesthesia, regional or sedation) is multifactorial, formulated around clinicians' expertise, preference, habit, policies, practicalities and may also be influenced by other healthcare professionals and patients. ${ }^{4}$ While the autonomous nature of anaesthesia is partly unavoidable, the lack of standardisation and consistency in how anaesthetic techniques are defined, administered and reported complicates the 
interpretation of published evidence and planning of future RCTs. ${ }^{15}$

The Consolidated Standards of Reporting Trials (CONSORT) makes recommendations for improving the quality of reporting in clinical trials, and is endorsed by many journals. ${ }^{6}$ Although published in 1996, several reviews have established that reporting standards of RCTs relating to anaesthetic interventions remain poor and concluded that clinicians and researchers would benefit from more uniform reporting. ${ }^{5-9}$ The more recent CONSORT extension for Non-Pharmacological Treatments (CONSORT-NPT) recognises the complexity of non-pharmacological interventions (which includes anaesthesia) that comprise multiple interacting components. ${ }^{10} 11$ Specific additions include the need to fully describe interventions, and standardise and monitor their delivery (ie, intervention fidelity) during RCTs, to facilitate reproducibility and ensure that effective interventions can be successfully implemented in clinical practice. $^{10}$

\section{STUDY AIM}

Anaesthesia provides an example of a complex intervention that the CONSORT-NPT extension targets for improved reporting in RCTs. To date, no study has assessed the quality of reporting of anaesthesia in relation to CONSORT-NPT. The aim of this study, therefore, is to systematically review and summarise the reporting of anaesthesia as an intervention in RCTs.

\section{METHODS}

This systematic review protocol follows the Preferred Reporting Items for Systematic Review and Meta-Analysis Protocols (PRISMA-P) guidelines. ${ }^{12}$ This review protocol has been registered with the International Prospective Register of Systematic Reviews (PROSPERO), and the protocol will be updated with amendments if required.

\section{DATA SOURCES AND SEARCH STRATEGY}

We will employ a hand-search methodology similar to that previously described by Blencowe $e t$ al in a systematic review of intervention design and delivery in surgery. ${ }^{13}$

Online archives of selected journals will be systematically hand searched to identify relevant articles. Articles published in the top journals by impact factor as listed in the Scimago Journal \& Country Rank for anaesthesia $(n=6)$, general medicine $(n=3)$ and general surgery $(n=3)$ will be included (table 1). ${ }^{14-16}$ Thomson Reuters Journal Citation Reports: Web of Knowledge will be accessed to confirm impact factor by citation. A hand-searching methodology will be used whereby content pages of relevant journals will be screened to identify eligible articles, rather than undertaking a formal search using $\mathrm{MeSH}$ terms and text words.
Table 1 The top six Scimago Journal \& Country Rank for 'Anesthesiology and Pain Medicine' and the top three for 'Medicine' and 'Surgery' for all countries in 2018 by impact factor (excluded journals from this list include those specifically related to leukaemia, pain, neuromodulation, palliative and perioperative medicine)

\begin{tabular}{|c|c|}
\hline Anaesthesiology & 1. Anesthesiology \\
\hline and Pain & 2. British Journal of Anaesthesia \\
\hline & $\begin{array}{l}\text { 3. Regional Anesthesia and Pain } \\
\text { Medicine }\end{array}$ \\
\hline & 4. Anaesthesia \\
\hline & 5. European Journal of Anaesthesiology \\
\hline & 6. Anaesthesia and Analgesia \\
\hline Medicine & 1. New England Journal of Medicine \\
\hline & 2. The Lancet \\
\hline & 3. JAMA \\
\hline Surgery & 1. Annals of Surgery \\
\hline & 2. British Journal of Surgery \\
\hline & 3. JAMA Surgery \\
\hline
\end{tabular}

There will be no limit on country of study. Studies published from 1 January 2016 to 1 September 2019 written in English will be included. This time period was chosen following scoping work to ensure a sufficient number of RCTs will be included in the review.

\section{STUDY SELECTION INCLUSION AND EXCLUSION CRITERIA}

Full-text RCTs describing the use of anaesthetic interventions within any invasive procedure in humans will be included. Studies where techniques comparing anaesthesia in cadavers, laboratory and animal studies will be excluded.

We define invasive procedures as 'purposeful/deliberate access to the body is gained via an incision, percutaneous puncture, where instrumentation is used in addition to the puncture needle, or instrumentation via a natural orifice. It begins when entry to the body is gained and ends when the instrument is removed, and/ or the skin is closed. Invasive procedures are performed by trained healthcare professionals using instruments, which include, but are not limited to, endoscopes, catheters, scalpels, scissors, devices and tubes' ${ }^{17}$ All modes of anaesthesia and anaesthesia techniques will be included. This will include studies comparing different modes of anaesthesia (eg, general, local, regional anaesthesia or sedation), as well as studies comparing different types/ techniques of the same mode of anaesthesia (volatile vs intravenous GA).

Case reports, non-randomised studies, retrospective and other non-comparative studies will be excluded. Abstracts and conference proceedings will be excluded due to high probability of incomplete data. The hand-search strategy is shown in online supplementary appendix 1 . 


\section{STUDY MANAGEMENT}

Electronic article information will be downloaded into EndNote software. Abstracts and titles will be screened independently by two researchers to identify articles that are potentially relevant, for which the full-text articles will be obtained. Full-text articles will be screened against the inclusion criteria by one researcher.

\section{DATA EXTRACTION AND ASSESSMENT}

Data will be extracted using a prespecified form in keeping with the standard CONSORT checklist for reporting trials. Study data will be collected and managed using REDCap electronic data capture tools hosted at the University of Bristol, UK. ${ }^{18}{ }^{19}$ Data extraction will be completed by one researcher and verified by a second independent researcher. Disagreements will be resolved through discussion with the senior researcher/other members of the research team.

The country of study, number and type of included centres and the volume of invasive procedures undertaken in each setting will be recorded.

Descriptions of the following study demographics will be recorded: journal of publication, anaesthetic mode and subtype as stated in the paper, availability of study protocol and, if available, the format of the protocol (weblink, published article or obtained through contacting authors directly).

The type of anaesthetic intervention described in each study will be recorded in keeping with the CONSORT-NPT checklist in as much detail as is published in either the included study or extracted study protocol.

The anaesthetic intervention will be recorded according to an initial draft typology of anaesthetic interventions developed by the authors. Reporting of anaesthetic technique will be categorised according to whether it was performed pre-procedurally, intra-procedurally or postprocedurally. This will be to allow as much information as is included in each study to be recorded and subsequently categorised. All studies providing information about any aspect of the anaesthetic intervention will be classified as reporting a description, regardless of the included level of detail. Any citations to anaesthetic interventions within the studies will be recorded separately.

Any reference to standardisation with regard to any anaesthetic technique will be recorded, including how and why this was done, and to which standard. For the purpose of this review, standardisation will be defined as a process 'to establish a standard consisting of regulations for how something is to be done'. ${ }^{20}$

The invasive procedure for which the anaesthetic was being used will be recorded, but no details regarding how the invasive procedure was performed will be recorded.

\section{FIDELITY}

Fidelity will be defined as "how far those responsible for delivering an intervention actually adhere to the intervention as it is outlined by its designers' as previously described by Blencowe et al. ${ }^{13}$ For each study, the reporting of fidelity to the anaesthetic intervention will be recorded as per the CONSORT-NPT guideline. Assessment of fidelity will be performed through details of any strategies implemented in the study to improve fidelity and details of how it was measured. This will include any crossover between trial arms of participants.

\section{ASSESSMENT OF RISK OF BIAS}

The Cochrane Collaboration's revised risk of bias tool will be used to assess the internal validity of selected RCTs. ${ }^{21}$ The tool includes an assessment of sequence generation and allocation concealment, blinding of participants and outcome assessment, incomplete outcome data and selective outcome reporting. Given that no meta-analysis will be performed for this review, the risk of bias assessment will be reported as an appendix within the main review.

\section{DATA ANALYSIS}

A PRISMA flow chart of search and study selection with included and excluded studies will be presented. Reasons for exclusion of studies will be given. Extracted data will be presented in tables.

Descriptive statistics will be used to summarise general study details including types and modes of anaesthetic interventions and reporting standards of the trials. The reporting of anaesthetic interventions against the CONSORT-NPT checklist will be reported qualitatively and in tabulated form. The reporting of anaesthetic interventions for the anaesthesia and non-anaesthesia journals will be reported both in combination and separately, to examine reporting standards between these journal types.

Formal statistical comparisons will not be undertaken in this review as the aim is to summarise reporting standards and not analyse specific trial results. This is in keeping with published systematic reviews that have summarised reporting standards in other research fields. ${ }^{132}$

\section{PATIENT AND PUBLIC INVOLVEMENT}

There was no involvement of patients or the public in the design of this systematic review, and the research question was not formulated or developed with patient or public involvement. Additionally, no patients or public members will be required in order to complete the systematic review.

Twitter Natalie S Blencowe @NatalieBlencowe, Sethina Watson @morefluids and Robert J Hinchliffe @robhinchliffe1

Contributors LE contributed to conception and design of the study, has written and reviewed the manuscript before submission. KC, NSB, RM and RJH contributed to conception and design of the study and have reviewed the manuscript before submission. MQ and SW have reviewed the manuscript before submission. All authors have given final approval for publication and agree to be accountable for all aspects of the work. 
Funding This work was undertaken with the support of the NIHR Biomedical Research Centre at University Hospitals Bristol NHS Foundation Trust and the University of Bristol, the Association of Anaesthetists of Great Britain and Ireland (AAGBI)/National Institute of Academic Anaesthesia (NIAA) grant and the University of Bristol Elizabeth Blackwell Clinical Primer Scheme.

Disclaimer The views expressed in this publication are those of the author(s) and not necessarily those of the NHS, the National Institute for Health Research or the Department of Health and Social Care.

Competing interests None declared.

Patient consent for publication Not required.

Provenance and peer review Not commissioned; externally peer reviewed.

Open access This is an open access article distributed in accordance with the Creative Commons Attribution Non Commercial (CC BY-NC 4.0) license, which permits others to distribute, remix, adapt, build upon this work non-commercially, and license their derivative works on different terms, provided the original work is properly cited, appropriate credit is given, any changes made indicated, and the use is non-commercial. See: http://creativecommons.org/licenses/by-nc/4.0/.

\section{ORCID iDs}

Lucy Elliott http://orcid.org/0000-0002-4265-4075

Karen Coulman http://orcid.org/0000-0003-0510-4290

Natalie S Blencowe http://orcid.org/0000-0002-6111-2175

Ronelle Mouton http://orcid.org/0000-0001-9562-8199

Robert J Hinchliffe http://orcid.org/0000-0002-6370-0800

\section{REFERENCES}

1 Armstrong RA, Mouton R. Definitions of anaesthetic technique and the implications for clinical research. Anaesthesia 2018;73:935-40.

2 American Society of Anesthesiologists: Quality Management and Departmental Administration Committee. Continuum of depth of sedation: definition of general anesthesia and levels of sedation/ analgesia, 2014. Available: www.asahq.org/quality-and-practicemanagement/practice-guidance-resource-documents/continuum-ofdepth-of-sedation- definition-of-general-anesthesia-and-levels-ofsedation-analgesia [Accessed 16th June 2019].

3 Royal College of Anaesthetists. Guidelines for the provision of anaesthetic services, 2016. Available: www.rcoa.ac. uk/documentstore/guidance-the-provi sion-of-sedation-services-2016 [Accessed 22 Jul 2019].

4 Dooley J, Armstrong RA, Jepson M, et al. Qualitative study of clinician and patient perspectives on the mode of anaesthesia for emergency surgery. Br J Surg 2019. doi:10.1002/bjs.11243. [Epub ahead of print: 01 Aug 2019].

5 O'Donnell CM, McLoughlin L, Patterson CC, et al. Perioperative outcomes in the context of mode of anaesthesia for patients undergoing hip fracture surgery: systematic review and metaanalysis. Br J Anaesth 2018;120:37-50.

6 Altman DG. Better reporting of randomised controlled trials: the CONSORT statement. BMJ 1996;313:570-1.

7 Shanthanna H, Kaushal A, Mbuagbaw L, et al. A cross-sectional study of the reporting quality of pilot or feasibility trials in high-impact anesthesia journals. Can J Anaesth 2018;65:1180-95.

8 Chow JTY, Turkstra TP, Yim E, et al. The degree of adherence to CONSORT reporting guidelines for the Abstracts of randomised clinical trials published in anaesthesia journals: a cross-sectional study of reporting adherence in 2010 and 2016. Eur J Anaesthesiol 2018:942-8.

9 Halpern SH, Darani R, Douglas MJ, et al. Compliance with the CONSORT checklist in obstetric anaesthesia randomised controlled trials. Int J Obstet Anesth 2004;13:207-14.

10 Boutron I, Moher D, Altman DG, et al. Extending the CONSORT statement to randomized trials of nonpharmacologic treatment: explanation and elaboration. Ann Intern Med 2008;148:295-309.

11 Boutron I, Altman DG, Moher D, et al. Consort statement for randomized trials of nonpharmacologic treatments: a 2017 update and a consort extension for nonpharmacologic trial Abstracts. Ann Intern Med 2017;167:40-7.

12 Moher D, Liberati A, Tetzlaff J, et al. Preferred reporting items for systematic reviews and meta-analyses: the PRISMA statement. BMJ 2009;339:b2535.

13 Blencowe NS, Boddy AP, Harris A, et al. Systematic review of intervention design and delivery in pragmatic and explanatory surgical randomized clinical trials. Br J Surg 2015;102:1037-47.

14 Scimago. Scimago Journal \& Country Rank; Medicine; Anaesthesia and Pain Rankings, 2018. Available: https://www.scimagojr.com/ journalrank.php?area=2700\&category=2703 [Accessed 16 Jun 2019].

15 Scimago. Scimago Journal \& Country Rank; Medicine; Medicine, 2018. Available: https://www.scimagojr.com/journalrank.php?area= 2700\&category $=2701$ [Accessed 16 Jun 2019].

16 Scimago. Scimago Journal \& Country Rank; Medicine; Surgery, 2018. Available: https://www.scimagojr.com/journalrank.php?area= 2700\&category=2746 [Accessed 6th January 2020].

17 Cousins S, Blencowe NS, Blazeby JM. What is an invasive procedure? A definition to inform study design, evidence synthesis and research tracking. BMJ Open 2019;9:e028576.

18 Harris PA, Taylor R, Thielke R, et al. Research electronic data capture (REDCap)--a metadata-driven methodology and workflow process for providing translational research informatics support. J Biomed Inform 2009;42:377-81.

19 Harris PA, Taylor R, Minor BL, et al. The REDCap Consortium: building an international community of software platform partners. $J$ Biomed Inform 2019;95:103208.

20 Collins Dictionary. Available: https://www.collinsdictionary.com/about [Accessed 16 Jun 2019].

21 Sterne JAC, Savović J, Page MJ, et al. Rob 2: a revised tool for assessing risk of bias in randomised trials. BMJ 2019;2:14898.

22 Coulman KD, MacKichan F, Blazeby JM, et al. Patient experiences of outcomes of bariatric surgery: a systematic review and qualitative synthesis. Obes Rev 2017;18:547-59. 